\title{
Robotic nephrolithotomy and pyelolithotomy with utilization of the robotic ultrasound probe
}

\author{
Khurshid R. Ghani, Quoc-Dien Trinh, Wooju Jeong, Ariella Friedman, Yegapan Lakshmanan, Mani Me- \\ non, Jack S. Elder \\ Vattikuti Urology Institute, Henry Ford Health System (KRG, QDT, WJ, AF, MM, JSE) and Department \\ of Pediatric Urology, Children's Hospital of Michigan (YL, JSE), Detroit, MI, USA
}

\begin{abstract}
Introduction: The treatment of large renal stones in children can be challenging often requiring combination therapy and multiple procedures. The purpose of this video is to describe our technique of robotic nephrolithotomy and pyelolithotomy for complex renal stone disease in children, and to demonstrate the utility of the robotic ultrasound probe to aid with stone localization.

Materials and Methods: Robotic nephrolithotomy/pyelolithotomy was carried out in four consecutive patients. A robotic ultrasound probe (Hitachi-Aloka, Tokyo, Japan) under console surgeon control was used in all cases.

Results: Two patients underwent robotic pyelolithotomy, one patient underwent robotic nephrolithotomy, whilst the fourth patient underwent robotic pyelolithotomy and nephrolithotomy along with Y-V pyeloplasty for concurrent ureteropelvic junction obstruction. Mean operative time, blood loss and hospital stay was 216 minutes, $37.5 \mathrm{~mL}$ and 2 days, respectively. The robotic ultrasound probe aided identification of calculi within the kidney in all cases. For nephrolithotomy it was helpful in planning the incision for nephrotomy. After nephrotomy or pyelotomy, stones were removed using a combination of robotic Maryland forceps, fenestrated grasper or Prograsp. Antegrade nephroscopy introduced through a laparoscopic port was used in all patients for confirmation of residual stone status. Two patients did not require a ureteral stent in the post-operative period. One patient had a minor complication (Clavien Grade 2 - dislodged malecot catheter). All patients were stone free at last follow-up.

Conclusions: Robotic nephrolithotomy and pyelolithotomy with utilization of the robotic ultrasound probe offers a onestop solution for complex renal stones with excellent stone-free rates.
\end{abstract}

\section{ARTICLE INFO}

Available at: www.brazjurol.com.br/videos/january_february_2014/Ghani_125_126video.htm

Int Braz J Urol. 2014; 40 (Video \#2): 125-6

Submitted for publication:

December 01, 2013

Accepted after revision:

January 30, 2014
Correspondence address:

Khurshid R. Ghani MS FRCS Vattikuti Urology Institute Henry Ford Health System 2799 W. Grand Boulevard, Detroit, Michigan, 48202, USA Fax: +1 313 916-4352 E-mail address: krghani@gmail.com 


\section{EDITORIAL COMMENT}

The video by Ghani et al. nicely demonstrates the techniques of robotic pyelolithotomy and nephrolithotomy in children. The video highlights the use of intraoperative ultrasonography using a specially designed probe that can be manipulated by the operating surgeon. Newer probes

\section{REFERENCES}

1. Yakoubi R, Autorino R, Laydner H, Guillotreau J, White MA, Hillyer $S$, et al. Initial laboratory experience with a novel ultrasound probe for standard and single-port robotic kidney surgery: increasing console surgeon autonomy and minimizing instrument clashing. Int J Med Robot. 2012; 8: 201-5.

2. Gill IS, Desai MM, Kaouk JH, Meraney AM, Murphy DP, Sung GT, et al.: Laparoscopic partial nephrectomy for renal tumor: duplicating open surgical techniques. J Urol. 2002; 167 (2 Pt 1): 469-7; discussion 475-6. allow the surgeon to directly control the probe with standard robotic instruments (1). Intraoperative ultrasonography has proven useful during partial nephrectomy for tumor identification to facilitate complete resection $(2,3)$. It is useful in parenchymal incision planning. It is especially helpful in difficult cases such as the completely intraparenchymal tumor (4).

3. Fazio LM, Downey D, Nguan CY, Karnik V, Al-Omar M, Kwan K, et al:: Intraoperative laparoscopic renal ultrasonography: use in advanced laparoscopic renal surgery. Urology. 2006; 68: 723-7.

4. Chung BI, Lee UJ, Kamoi K, Canes DA, Aron M, Gill IS: Laparoscopic partial nephrectomy for completely intraparenchymal tumors. J Urol. 2011; 186: 2182-7.

Hubert Swana, MD Pediatric Urology Nemours Children's Hospital Orlando Orlando, FL, USA E-mail: hswana@nemours.org 